\title{
Dislocation nucleation and evolution at the ferrite-cementite interface under cyclic loadings
}

\author{
Lun-Wei Liang, ${ }^{\mathrm{a}, \mathrm{b}}$, Yun-Jiang Wang ${ }^{\mathrm{a}, \mathrm{b}, *}$, Yan Chen ${ }^{\mathrm{a}, \mathrm{b}}$, Hai-Ying Wang ${ }^{\mathrm{a}, \mathrm{b}}$, Lan-Hong Dai ${ }^{\mathrm{a}, \mathrm{b}, *}$ \\ a State Key Laboratory of Nonlinear Mechanics, Institute of Mechanics, Chinese Academy of Sciences, Beijing 100190, PR China \\ ${ }^{\mathrm{b}}$ School of Engineering Science, University of Chinese Academy of Sciences, Beijing 101408, PR China
}

\section{A R T I C L E I N F O}

\section{Article History:}

Received 24 August 2019

Revised 9 December 2019

Accepted 27 December 2019

Available online 6 January 2020

\section{Keywords:}

Pearlitic steels

Cyclic deformation

Mechanical property

Interfacial dislocation

Molecular dynamics

\begin{abstract}
A B S T R A C T
Fatigue is of significant importance to the engineering applications of the structural materials. High-strength pearlite steel consisting of a ductile ferrite phase and a brittle cementite phase is a widely used structural metal for extreme load-bearing applications. However, the fatigue mechanisms of such important materials remain elusive, in particular, the atomic-scale dislocation behaviors at interface are poorly understood. We used molecular dynamics simulations to probe the mechanical response and deformation mechanism of the Bagaryatskii-oriented ferrite-cementite interface in pearlite. The interface was subjected to a hundred symmetric tension-compression deformation cycles. Three different loading schemes with strain magnitudes of 4.0\%, 6.0\%, and 9.0\% are sophisticatedly designed to explore the cyclic plastic mechanisms under different conditions corresponding to pure elasticity, elasticity in tension but plasticity in compression, and plasticity in both tension and compression, respectively. During cyclic deformation, rapid dislocation accumulation occurs in the first 30 cycles, after which dislocation density decreases to a stable value in ferrite. It is found that the onset of plasticity is governed by dislocation nucleation from the ferrite-cementite interface. After slip into the ferrite phase, some dislocations annihilate at the interface. After a few tens of cycles, the dislocation nucleation and annihilation rates become equal, leading to a steady-state flow in cyclic deformation. Up to high cycles with large strain magnitude, the magnitude of plastic strain in pearlite is higher than critical values and slip crosses the interface from the ferrite phase to the brittle cementite phase. Dislocation slip in cementite will destroy the interface structure, which may be the plastic mechanism of final fatigue failure. Our simulations agree with experimental observations of dislocation evolution in the ratchetting of pearlitic steels and provide further atomic-scale mechanisms to explain the fatigue failure of these materials.
\end{abstract}

(C) 2020 Acta Materialia Inc. Published by Elsevier Ltd. All rights reserved.

\section{Introduction}

Cold-drawn pearlitic steel wires are used widely in engineering structures such as automobile tires, suspension bridge cables, and engineering springs because they combine high strength and reasonable ductility [1]. Pearlite, which is derived from the eutectic transformation of austenite, consists of alternating lamellae of body-centered cubic (BCC) ferrite $\left(\alpha\right.$-Fe) and orthorhombic cementite $\left(\mathrm{Fe}_{3} \mathrm{C}\right)$. Fatigue due to high-amplitude cyclic stress is the primary cause of mechanical failure in structural components, accounting for approximately $90 \%$ of metallic failures [2,3]. In practical service conditions, pearlitic steel structures such as mine hoists and tower cranes are inevitably subjected to ratchetting deformation [4], which is a cyclic accumulation of inelastic deformation generated

\footnotetext{
* Corresponding authors at: State Key Laboratory of Nonlinear Mechanics, Institute of Mechanics, Chinese Academy of Sciences, Beijing 100190, PR China and School of Engineering Science, University of Chinese Academy of Sciences, Beijing 101408, PR China

E-mail addresses: yjwang@imech.ac.cn (Y.-J. Wang), lhdai@Inm.imech.ac.cn (L.-H. Dai).
}

by asymmetric stress-controlled cyclic loading. However, the exact pathway of plastic strain accumulation and the failure mechanism under cyclic deformation is poorly understood. Therefore, it is critical to explore the mechanism underlying cyclic deformation, in particular down to atomic scale.

The evolution of various configurations of dislocations and their evolution behaviors strongly influences the microscopic mechanisms underlying cyclic plastic deformation in metallic materials. Gaudin and Feaugas have found that a threshold stress induces the formation of two types of dislocation trapping in AISI 316L stainless steels subjected to ratchetting processes [4]. A transformation of the dislocation configuration from lowdensity lines and tangles to high-density cells and sub-grains during ratchetting deformation has been observed in polycrystalline 20 ordinary carbon steels (BCC) [5] and 316L stainless steels (face-centered cubic, FCC) [6] by using transmission electron microscopy (TEM) observations. Recently, Liang et al. have used TEM and Mössbauer spectrometry to examine the effects of the interactions between dislocations and decomposed carbon atoms from cementite on the strengthening and plastic mechanisms underlying the ratchetting deformation of cold-drawn 
pearlitic steel wires [7]. However, the exact deformation mechanisms in pearlite under cyclic deformation still remain unclear because of the limitations of spatial-temporal resolution in the experimental characterization techniques.

Furuhara et al. have characterized systemically the effect of the interfaces on the mechanical properties of steels [8-10]. The ferritecementite interface plays a critical role in the mechanical behaviors of pearlite because interface serves as either source and sink, or block of dislocations. The resultant plastic deformation mechanisms also include interface crossings [11-13], confined layer slips [14], and dislocation pile-ups $[15,16]$. Numerous atomistic modeling studies have concentrated on the energies and structures of ferrite-cementite interfaces up to now. In pearlite, different atomic termination planes of the cementite supercell at the ferrite-cementite interface dictate the chemistry and registry of the interface, which eventually determine the interfacial energies [17-19]. Dislocation arrays are formed at the interfaces for all five orientation relationships (ORs), i.e., Bagaryatskii et al. [20], Isaichev, Pitsch-Petch, Near-Bagaryatskii, and Near-Pitsch-Petch as reported in the literatures $[17,19]$. An extended atomically informed Frank-Bilby (xAIFB) method [21] and a continuum model $[17,19]$ have been proposed to characterize the structures of misfit dislocations. They are capable of quantitatively predicting dislocation information (dislocation vectors and spacing) and interfacial energies, respectively. Meanwhile, atomistic modeling studies have also been used to investigate the mechanical properties of ferrite-cementite interfaces with the Bagaryatskii orientation relationship (OR) under monotonic uniaxial tensile and compressive deformation. Guziewski et al. have noted that the mechanical properties of the ferrite-cementite interface strongly depend on the lamellar thickness, on the volume ratios, and on the various ORs between the ferrite and cementite phases [22,23]. More recently, Shimokawa et al. have reported that higher ductility can be achieved in drawn pearlite by controlling the interfacial dislocation spacing. The spacing accommodates the misfit strain between the ferrite and cementite phases, and consequently determines the phase stress and the interfacial dislocation structure [24]. We have found in previous work [25] that the increased strength and ductility of annealed pearlite is caused by the decomposed carbon atoms, which block the movement of dislocations. Annealing also leads to non-continuous cementite layers that can serve as tunnels for the spread of dislocations and improve the ductility. Finally, Ghaffarian et al. have reported that the ductility of pearlite is related to the loading direction and is improved by either increasing the temperature or reducing the interlamellar spacing [26].

Existing atomistic modelings focus mostly on the static interface structures and energetics and on the interface dislocation structure and evolution under monotonic loading. The plastic response of the ferrite-cementite interface under cyclic deformation, which is more relevant to the fatigue mechanism of pearlitic steels, is less frequently discussed.

In the present work, we settle the issues by characterizing the roles of interfaces and interfacial dislocations in the deformation behavior of the nanoscale lamellar between the ferrite and cementite phases in pearlite. The interface with Bagaryatskii OR under symmetric tension-compression cyclic deformation was simulated by using molecular dynamics (MD). In Section 2 we describe the atomistic model and calculation details. Simulations were also performed to obtain the fully relaxed structure of the ferrite-cementite interface. Monotonic uniaxial tensile and compressive tests were carried out to obtain the mechanical properties. Three different strain levels were chosen for cyclic deformation with a constant strain rate. In Section 3 we present the cyclic stress response, dislocation density analysis, and dislocation evolution at the three different strain levels. At low strain (4.0\%), elastic deformation occurs in both ferrite and cementite phases and no interfacial dislocation nucleates from the ferritecementite interface. At an intermediate strain (6.0\%), only ferrite deforms plastically, while cementite deforms elastically. At high strain (9.0\%), plastic and inelastic deformation occurs in the ferrite and cementite phases, respectively. During cyclic plastic deformation (6.0\% and 9.0\%), dislocation density initially increases rapidly before decreasing and finally evolving to a steady state. In Section 4 we analyze the mechanism underlying cyclic deformation in pearlite. We capture the process of dislocation nucleation and annihilation during cyclic plastic deformation. Slip transfer through the ferrite-cementite interface is characterized to study the synergistic effect between the ferrite and cementite phases. To the best of our knowledge, the current work is possibly the first attempt to explore the fatigue mechanism in pearlitic steels at the atomic level. Our simulations shed light on the cyclic mechanical response and fatigue mechanism of pearlitic steels by elucidating the interplay between the dislocations and the interfaces. It also allows for the comparison of interface with other ORs, as well as cementite decomposition process in the near future.

\section{Methodology}

\subsection{Atomic model}

MD simulations were performed using the Large-scale Atomic/ Molecular Massively Parallel Simulator (LAMMPS) package [27]. Although five orientation relationships have been reported so far, the Bagaryatskii OR is the most widely studied for its high degree of registry. Using the convention $a \leq b \leq c$ for the unit cell of cementite, the Bagaryatskii OR can be expressed as: $[100]_{\theta}\left\|[1 \overline{1} 0]_{\alpha},[010]_{\theta}\right\|[111]_{\alpha}$ and $(001)_{\theta} \|(11 \overline{2})_{\alpha}$. Here the subscripts $\theta$ and $\alpha$ denote cementite and ferrite, respectively. We used the Tersoff potential developed by Henriksson et al. [28] to maintain a balance between computation cost and accuracy. The calculated lattice parameters of orthorhombic cementite are $a=4.48 \AA, b=4.96 \AA$, and $c=6.47 \AA$, which are in good agreement with the experimental values of $a=4.52 \AA, b=5.08 \AA$, and $c=6.73 \AA$ [29]. The BCC Fe has a calculated lattice parameter of $a=2.89 \AA$, which is close to the experimental value of $a=2.87 \AA$ [30].

The simulation model has a "sandwiched" shape, with dimensions $L_{x} \times L_{y} \times L_{z}=13.1 \times 46.0 \times 14.2 \mathrm{~nm}^{3}(\sim 0.88$ million atoms $)$ oriented along the principal $x:[100]_{\theta}\left\|[1 \overline{1} 0]_{\alpha}, y:[010]_{\theta}\right\|[111]_{\alpha}$, and $z$ : $[001]_{\theta}$ \|l $[\overline{1} 12]_{\alpha}$ directions, as shown in Fig. 1 . The interfacial plane was made large enough to minimize the strain required to create a coherent interface $\left(L_{x} \times L_{y}=13.1 \times 46.0 \mathrm{~nm}^{2}\right)$. The strain is determined by $\varepsilon=2\left(L_{i}^{\theta}-L_{i}^{\alpha}\right) /\left(L_{i}^{\theta}+L_{i}^{\alpha}\right)(i=x, y)$, where $L_{i}^{\theta}$ and $L_{i}^{\alpha}$ represent the interfacial lengths of the cementite and ferrite phases, respectively. In this way, the strains in the $x$ and $y$ directions are calculated to be $-0.07 \%$

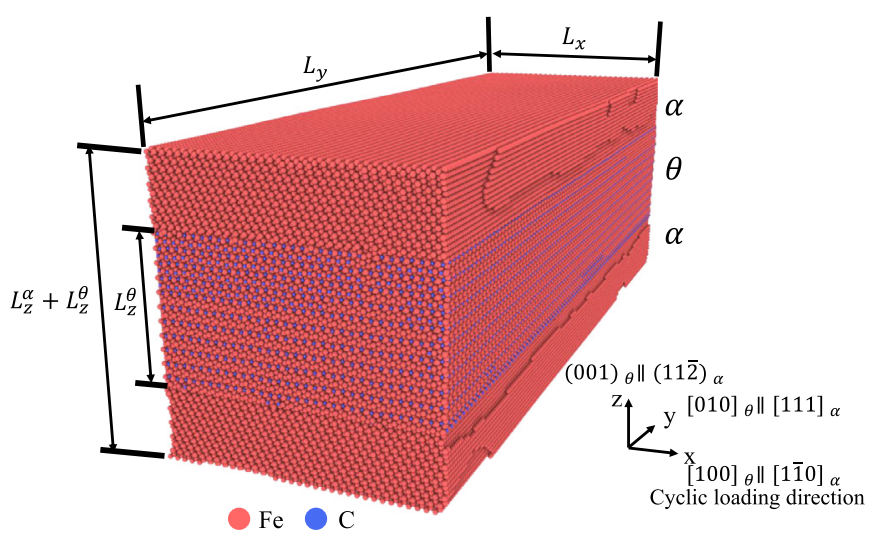

Fig. 1. Atomic model of the nanoscale lamellar structure with Bagaryatskii orientation ferrite-cementite interface, in which $\alpha$ and $\theta$ indicate the ferrite and cementite phases, respectively. Red and blue atoms represent Fe and carbon atoms, respectively. (For interpretation of the references to color in this figure legend, the reader is referred to the web version of this article.) 
and $0.24 \%$, respectively. The influence of different atomic terminating planes (Fe-FeC, FeC-Fe, and Fe-Fe) of cementite with the Bagaryatskii $\mathrm{OR}$ on the energetics and structures of the ferrite-cementite interface has been characterized by Guziewski et al. [19]. In the present work, both ferrite-cementite interfaces are made of the same Fe-FeC terminating plane to avoid carbon atoms in accordance with the scheme of Shimokawa et al. [24]. There are minimal differences in the mechanical response of the various terminating planes according to Refs. $[23,24]$. The cementite phase accounts for about one-ninth of the total volume in real eutectoid pearlite specimens. The focus of this work is on the plastic mechanisms of the ferrite-cementite interface under cyclic loadings. We applied a 1:1 ratio cementite-ferrite interface model to trace the mechanisms which saves computational cost. The ferrite slip systems are the major factors in determining which slip system in cementite will be activated [22]. As demonstrated in Ref. [24], the initially activated inelastic-deformation modes in ferrite, which are model S1 in tension and model S2 in compression, are the same for a near-perfect semi-coherent interface $\left(p_{1}^{-1}=0.25 \mathrm{~nm}^{-1}\right.$ and $p_{2}^{-1}=0.125 \mathrm{~nm}^{-1}$ ) with $1: 1$ and $8: 1$, respectively, ferrite to cementite volume ratios [24]. The only difference in models with various layer-thickness ratios is that the effective critical resolved shear stresses of the composite change [24]. But the plastic mechanisms remain unchanged. It suggests that the models with the near-perfect semi-coherent interface do show the same plastic mechanisms. Various layer-thickness-ratios only affect the activated time (or the magnitude of cyclic strain) of the onset of plasticity on the interface.

\subsection{Molecular statics and molecular dynamics}

Periodic boundary conditions were applied in all three directions. Molecular statics energy minimization was carried out at $0 \mathrm{~K}$ and zero pressure by using the conjugate gradient algorithm, after which MD annealing was performed. The simulated structure was assigned a temperature of $800 \mathrm{~K}$ and further equilibrated thermally at $800 \mathrm{~K}$ for 200 ps by using the Nosé-Hoover [31,32] isothermal-isobaric (NPT) ensemble [33]. Here $N, P$, and $T$ denote number of atoms, pressure, and temperature, respectively. After that, the system was cooled down to $5 \mathrm{~K}$ within $100 \mathrm{ps}$. The sample was then further minimized by using the conjugate gradient algorithm to obtain the fully relaxed structure, simultaneously by a thorough relaxation of stress tensor.

To ensure the robustness of the simulation results, the interfacial energies of three different terminating planes of cementite were calculated. The interfacial energies were calculated by introducing a vacuum region normal to the interface in consistent with the methods in our [25] and other [19] previous work. The interfacial energy of the system is defined as $\gamma_{\text {int }}=\frac{E_{\text {total }}-E_{\alpha}-E_{\theta}}{l_{x} l_{y}}-\gamma_{\alpha}-\gamma_{\theta}$, where $l_{x}$ and $l_{y}$ are the dimensions of the interface along the $x$ and $y$ directions, respectively. $\gamma_{\alpha}$ and $\gamma_{\theta}$ are the surface energies of ferrite and cementite in the normal dimensions, as shown in Fig. 1. $E_{\text {total }}, E_{\alpha}$, and $E_{\theta}$ are the total energies of the ferrite-cementite system, the bulk energy of the BCC ferrite, and the bulk energy of the cementite crystal, respectively. The interfacial energies for the $\mathrm{FeC}-\mathrm{Fe}, \mathrm{Fe}-\mathrm{FeC}$, and $\mathrm{Fe}-\mathrm{Fe}$ terminating planes are calculated to be $0.88,1.21$, and $2.19 \mathrm{~J} / \mathrm{m}^{2}$, respectively. These values are in excellent agreement with those in Ref. [19].

\subsection{Loading conditions and atomistic structural analysis}

For cyclic as well as monotonic tests, periodic boundary conditions are also applied along all three dimensions. Experiments have shown that the ferrite phase in cold-drawn pearlitic steels takes the $<110>$ fiber texture along the drawing direction [15,34]. To mimic the experimental conditions, the simulated structure was strained along the ferrite $[1 \overline{1} 0]$ direction $\left(L_{x}\right.$ axis in Fig. 1$)$ at a constant strain rate of $10^{9} \mathrm{~s}^{-1}$, while the other two dimensions $\left(L_{y}\right.$ and $L_{z}$ axes in Fig. 1) were left to relax freely to maintain a stress-free condition. The MD timestep was $2 \mathrm{fs}$, and the temperature was maintained at $5 \mathrm{~K}$ to minimize thermal noise. This allowed displacive mechanisms such as dislocations to predominate over diffusive mechanisms. The deformation was carried out under strain control.

Prior to fatigue tests, monotonic tension and compression were performed to determine the tensile and compressive properties, which are useful for establishing the strain levels to be applied under cyclic deformation. Fig. 2a shows the stress-strain curves under monotonic uniaxial tensile and compressive tests. The stress first increases to a certain peak stress and then drops steeply to a decreased flow stress. Similar results were observed during monotonic tensile and compressive deformation of the ferrite-cementite interface in Refs. [23-26]. The peak stress occurs because of the onset of plasticity. The tensile yielding stress ( $\sim 23.37 \mathrm{GPa})$ is higher than the compressive yielding stress ( $19.01 \mathrm{GPa})$, which is consistent with the results reported in Refs. [23,24]. This is due to different slip systems being activated under tension and compression, as shown in Supplementary Fig. S1. The $\{112\}<111>$ slip system with a Schmid factor of 0.47 is activated under tension, while the $\{110\}<111>$ slip system with a value of 0.41 is activated under compression, which are consistent with the findings in Ref. [24]. The critical strains corresponding to the compressive and tensile yielding stresses are $\sim 5.8 \%$ and $\sim 8.8 \%$, respectively. The simulated structure initially deforms plastically under compressive deformation (shown in Fig. 2a) because the magnitude of the residual Burgers vector under compressive deformation becomes smaller than that of the original interfacial dislocation [24].

We conducted three sophisticated conditions for symmetrical tension-compression cyclic tests up to different maximum strains in
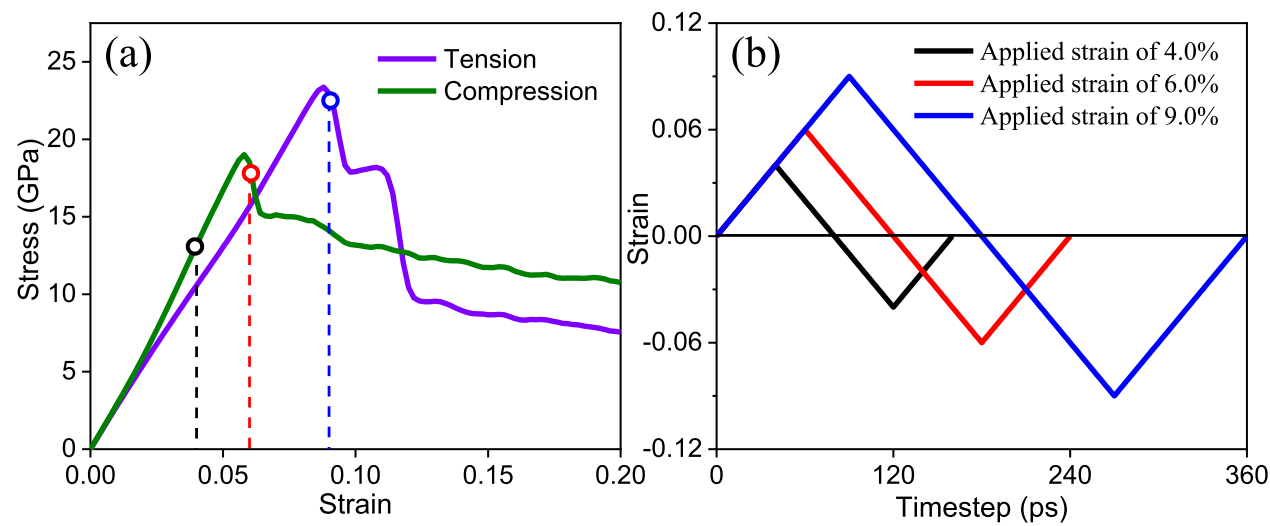

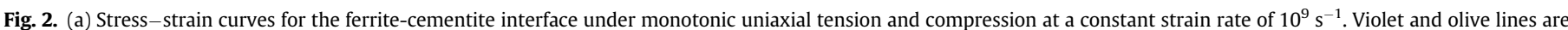

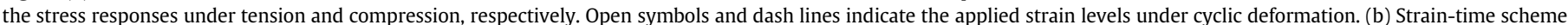

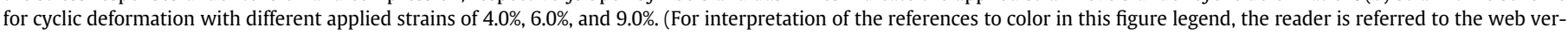
sion of this article.) 
each cycle $(4.0 \%, 6.0 \%$, and $9.0 \%)$. The first strain condition allows elastic deformation in the simulated system under both tensile and compressive cyclic deformation. The second strain condition yields elastic deformation in tension, but plastic deformation in compression. The third strain condition produces plastic deformation in both tension and compression. A strain-controlled triangular loading waveform was used in this cyclic study. Fig. 2b shows the typical strain-time scheme for the cyclic tests at each of the applied strain levels. Note that if not otherwise specified, positive and negative strains in this work represent tensile and compressive loading, respectively. The sign of the stress in cyclic deformation follows the same convention.

The OVITO software package [35] was used to visualize the different defects in the system. Dislocations and their characteristics were analyzed by using the dislocation extraction algorithm (DXA) tool [36]. The DXA method identifies all types of dislocations in common crystals (BCC, FCC, and hexagonal close-packed), determines their Burgers vectors, and outputs a line representation of the dislocation fragments. Common neighbor analysis (CNA), which computes a fingerprint for pairs of atoms, is also used to classify atoms in the crystallographic structure [37]. Atomic strain in the protocol of the von Mises local shear invariant was also calculated by using OVITO to analyze the strain accumulation process during cyclic deformation [38,39].

\section{Results}

\subsection{Cyclic stress response}

Cyclic stress was calculated to investigate the fatigue behavior of the ferrite-cementite interface within pearlite. The stress amplitude was computed as $\sigma_{\mathrm{a}}=\left(\sigma_{\max }-\sigma_{\min }\right) / 2$, where $\sigma_{\max }$ and $\sigma_{\min }$ are the maximum and minimum stress in each cycle, respectively [40]. Fig. 3 shows the stress-cycle responses and the amplitude stresscycle number plot at applied strains of $4.0 \%, 6.0 \%$, and $9.0 \%$. One hundred cycles were sufficient for the stress response to reach a steady state under all three strain conditions. Fig. 3a shows that $\sigma_{\max }$ and $\sigma_{\text {min }}$ remain unchanged under cyclic deformation at an applied strain of $4.0 \%$, meaning that both ferrite and cementite phases deform elastically. Fig. $3 \mathrm{~b}$ shows that $\sigma_{\text {min }}$ first decreases and then stabilizes, while $\sigma_{\max }$ remains unaltered at an applied strain of $6.0 \%$. This indicates that plastic deformation occurs in the compressive phase, while elastic deformation remains in the tensile phase. Fig. 3c shows that both $\sigma_{\max }$ and $\sigma_{\min }$ simultaneously decrease in the first few cycles and then reach a steady stage at an applied strain of 9.0\%, which suggests that plastic deformation appears in both tensile and compressive phases. Cyclic softening, defined as a decrease in stress amplitude under cyclic straining [41], is observed under cyclic deformation at applied strains of $6.0 \%$ and $9.0 \%$, as shown in Fig. 3 d. This is ascribed to the transition from dislocation nucleation plasticity at the ferrite-cementite interface to the motion of existing dislocations in the ferrite phase.

\subsection{Dislocation mechanism underlying cyclic deformation}

Low-cycle fatigue involves plastic deformation which is controlled by the formation and movement of dislocations [42]. Cementite is brittle and hard at room temperature due to covalent bonding, and is therefore difficult to deform plastically [43]. It is usually quite free of dislocations and sub-boundaries [44,45]. As a result, the majority of the dislocations exist in the ferrite phase. We therefore calculated quantitatively the dislocation density in ferrite for the three different cyclic loading conditions. Fig. 4a shows the dislocation density as a function of cycle number for the first two cycles. When the applied strain is $4.0 \%$, the dislocation density in ferrite is zero and no dislocation nucleates at the interface, indicating that the deformation in both ferrite and cementite phases is totally elastic. Fig. 4a shows that for an applied strain of $6.0 \%$ dislocation begins to nucleate at interface when $3 / 4$ cycle of the first cycle (compressive stage) is completed, while for an applied strain of $9.0 \%$ dislocation begins to generate at interface at $1 / 4$ cycle of the first cycle (tension stage). These results are in excellent agreement with the stress responses under monotonic tensile and compressive deformation in Fig. 2a. Fig. 4a also shows the variation in dislocation density during each cycle. This is because the specific triangular shape of the straining protocol that caused the different levels of plastic deformation. The dislocation density at an applied strain of $9.0 \%$ is higher than that at an applied strain of $6.0 \%$, which suggests that more dislocations nucleate in ferrite at higher strain level. When the strain magnitudes are larger, more dislocations are activated, as shown in the inset of Fig. 4a. A rapid increase in dislocation density is observed in the first 30 cycles. This corresponds well to the experimental trend, in which dislocation density increases rapidly because of the initial increase in plastic strain in pearlitic steels during ratchetting deformation [7].

We calculated the dislocation density values at four points corresponding to the strain magnitudes of $0, \varepsilon_{\max }, 0$, and $\varepsilon_{\min }$ in each cycle. Here $\varepsilon_{\max }$ and $\varepsilon_{\min }$ are the maximum and minimum strain values in each cycle, respectively. Fig. 4 b shows the dislocation density as a function of cycle number over 100 cycles. The dislocation density increases in the first 30 cycles for applied strains of $6.0 \%$ and $9.0 \%$, which suggests that rapid dislocation nucleation and multiplication occurs in ferrite after cyclic deformation. The final dislocation density fluctuates around an approximately constant value under applied strains of $6.0 \%$ and $9.0 \%$. Under the condition of the applied strain of $6.0 \%$, the dislocation density appears to return to zero in the first 12 cycles, but they cannot annihilate completely at interface after 12 cycles after this number of cycles. It may suggest that there are possibly two different mechanisms of dislocation annihilation during cyclic loading. One is dislocation annihilation happens on the opposite interface, while the other is dislocation on the same interface where dislocation nucleates. However, dislocation does not annihilate completely during cyclic loading under the condition of the applied strain of $9.0 \%$. Note that the dislocation density value in the first two cycles in Fig. 4a appears to be higher than the corresponding value shown in Fig. $4 \mathrm{~b}$. This is because we only collected the dislocation density data at the middle and end of each cycle, potentially missing the maximum values that usually occur at $\sim 3 / 4$ of each cycle.

\subsection{Closeup of dislocation evolution}

In this section we provide the atomic-scale plastic mechanisms underlying the cyclic deformation of the ferrite-cementite interface. Fig. 5a shows that in the first cycle of deformation under an applied strain of $4.0 \%$, no dislocation appears at the ferrite-cementite interface. The deformation was therefore elastic. However, when the cyclic strain is increased to $6.0 \%$, the dislocations nucleate at the ferrite-cementite interface at $3 / 4$ of the way through the first cycle, in the compressive stage. The dislocations are mainly of the $1 / 2<111>$ full type. At a still larger strain magnitude of $9.0 \%$, dislocation nucleation begins at $1 / 4$ of the way through the first cycle, in the tensile stage.

Fig. 6 shows snapshots of dislocations at different cycle numbers during cyclic deformation. Figs. $6 \mathrm{a}$ and $\mathrm{b}$ show the evolution of dislocations under applied strains of $6.0 \%$ and $9.0 \%$, respectively. Fig. $6 \mathrm{c}$ shows the initial configuration without dislocations in the ferrite phase for comparison. Snapshots of dislocation evolution under applied strain of $4.0 \%$ are excluded in Fig. 6 because no dislocation exists at the interface. Figs. $6 \mathrm{a}$ and $\mathrm{b}$ also show that most of the dislocations in ferrite are $1 / 2<111>$ dislocations. The number of dislocations first increases with cycle number before decreasing and eventually reaching a steady state. The snapshots are in accord with the changes in dislocation density in Fig. 4b. Because of larger strain and stress levels at an applied strain of 9.0\%, some defect meshes are more easily formed in the ferrite phase, as shown in Figs. $5 \mathrm{c}$ and $6 \mathrm{~b}$. 

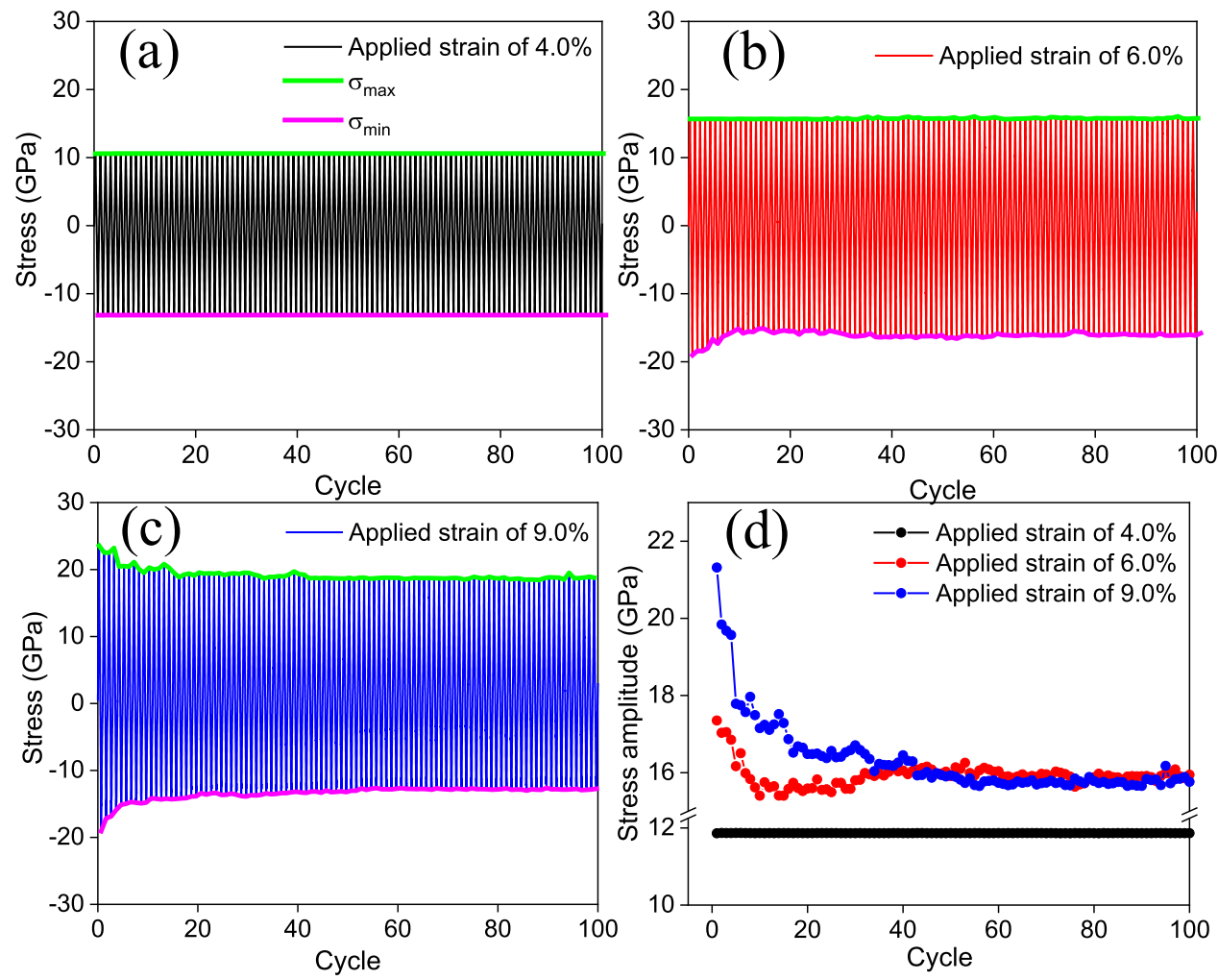

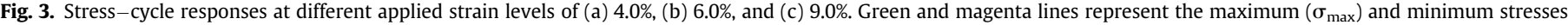

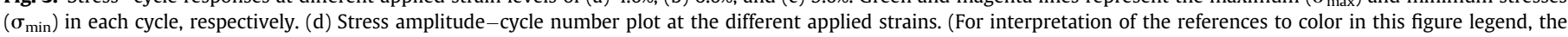
reader is referred to the web version of this article.)

Defect mesh, which encloses the non-dislocation defects, is a triangulated mesh which represents the bad crystal regions that have not been classified as dislocations [36]. In this work, it indicates the residual strains in the ferrite phase during cyclic deformation.

\section{Discussion}

\subsection{Increasing and decaying peak stress mechanisms}

At a low strain level of $4.0 \%$, the ferrite-cementite interface deforms purely elastically with constant peak stress amplitude and no dislocation nucleates at the interface. Elastic deformation occurs in both ferrite and cementite phases, which corresponds to an effectively infinite fatigue life without dislocation defects. This does not occur in practice because real-world pearlitic steels contain various types of impurities introduced during their production and in service. In addition, the surfaces of pearlitic steel wires are full of defects because of high friction with the molds used in the drawing process. These defects lead to local stress concentration that causes void formation during cyclic loading.

At a strain level of $6.0 \%$, the maximum stress $\left(\sigma_{\max }\right)$ remains unchanged because $\sigma_{\max }$ is lower than the critical stress for dislocation nucleation in the tensile stage. Therefore, no dislocation is activated during tension. The minimum stress $\left(\sigma_{\min }\right)$ in the compressive

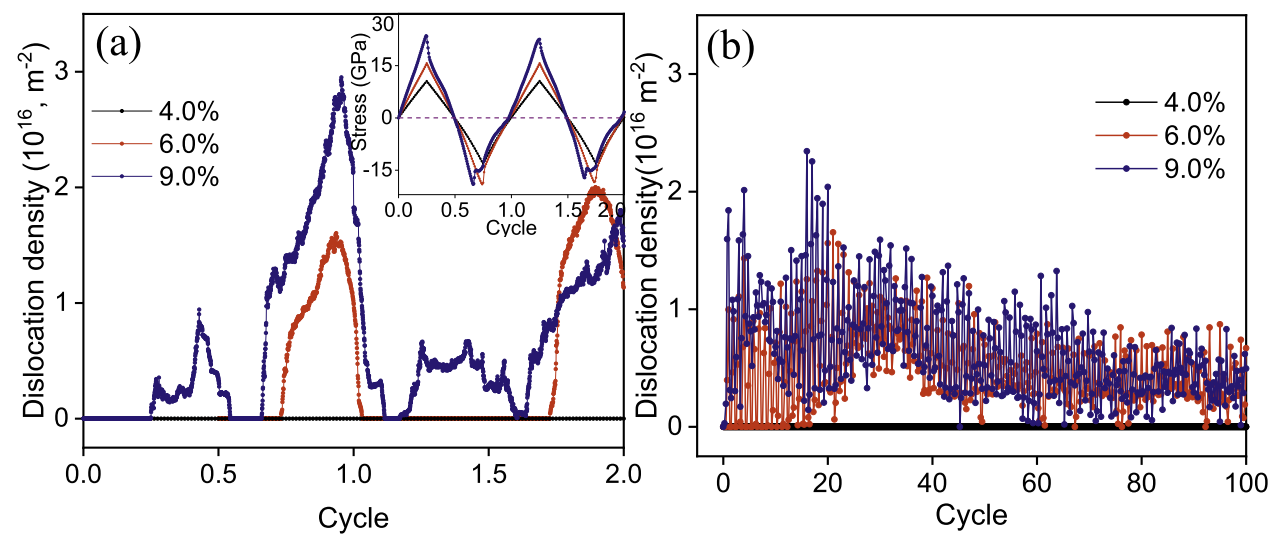

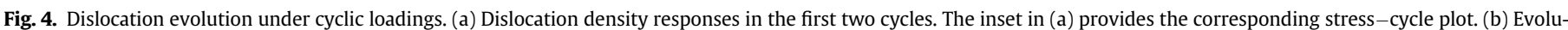

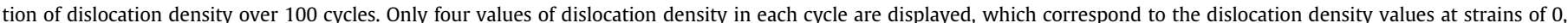

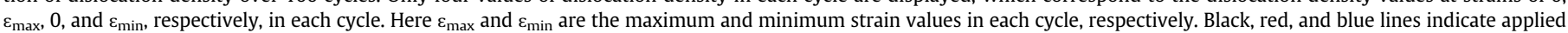
strains of $4.0 \%, 6.0 \%$, and $9.0 \%$, respectively. (For interpretation of the references to color in this figure legend, the reader is referred to the web version of this article.) 
(a)
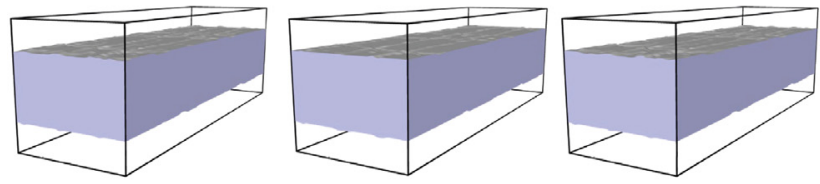

(b)

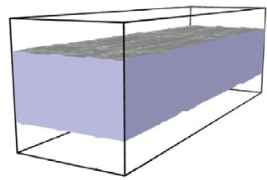

(c)

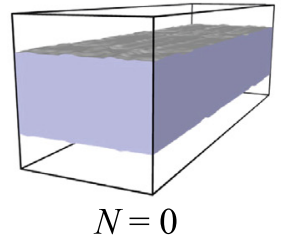

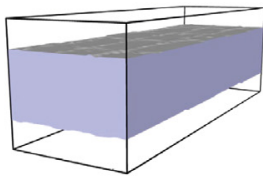
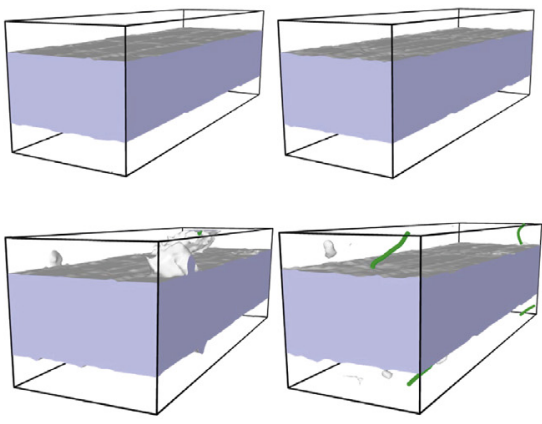

$N=1 / 4$

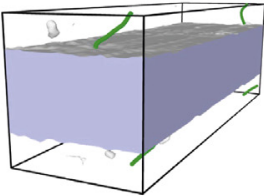

$N=2 / 4$
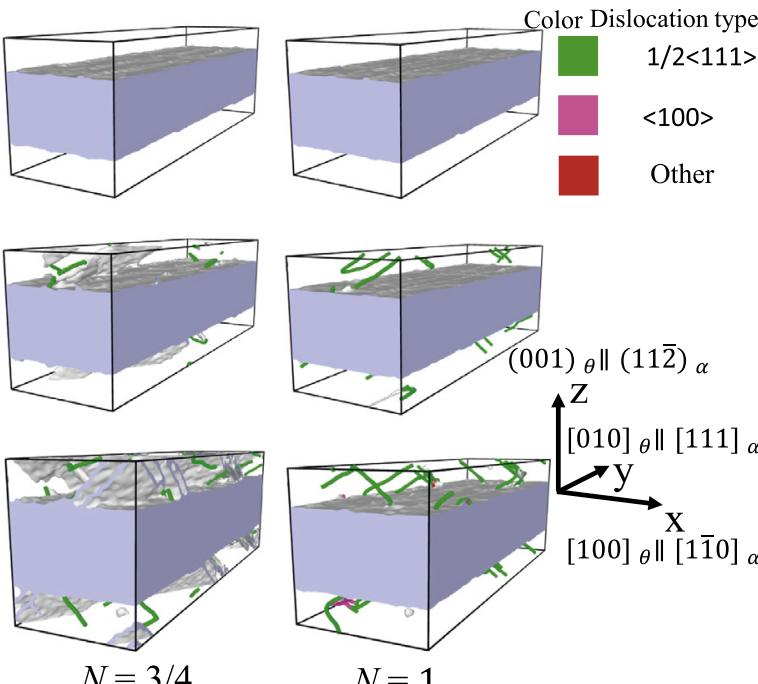

$N=3 / 4$
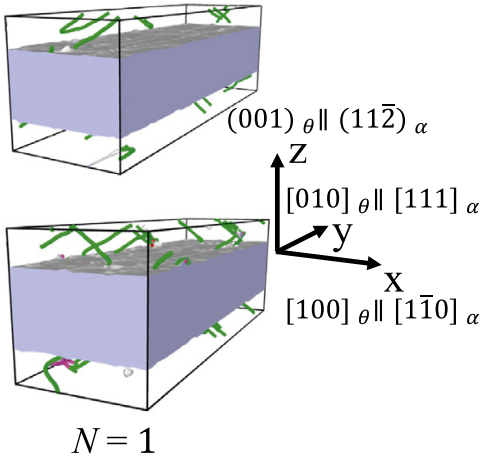

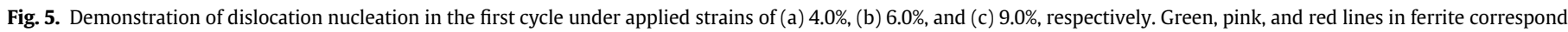

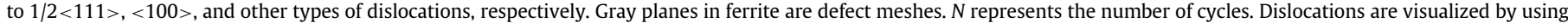

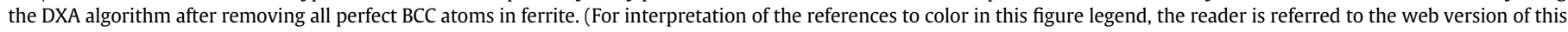
article.)

stage drops because dislocations initially nucleate at the interface for the first few tens of cycles, after which existing dislocations start to slip during subsequent cycles. At a high strain level of $9.0 \%$, the stress drops under both tension and compression. Under these conditions, dislocations nucleate and slip during both tensile and compressive stages. Cyclic softening (shown in Fig. 3d) is observed under cyclic deformation at high strain levels (6.0\% and $9.0 \%$ ) because of the transformation from dislocation nucleation to forest dislocation interactions including dislocation slip, multiplication, and annihilation. The stress requires to nucleate new dislocations at the interface is higher than the stress driving the plastic flow. Note that our simulation conditions are different from the experimental conditions in which colddrawn pearlitic steel wires [7] and BCC carbon steels [5] show work hardening with increasing cycle numbers under ratchetting deformation. The difference stems from the absence of initial dislocations, grain boundaries, and decomposed carbon atoms in ferrite in our simulations. The dislocation nucleation stage is therefore less significant in ratchetting deformation experiments [7].

\subsection{Slip transfer from ferrite to cementite}

Our simulations also give indication to the final fatigue and failure mechanism of pearlitic steels. The process of slip transmission across the interphase boundary is critical to the mechanical properties of pearlitic steels [46]. We calculated the atomic-scale equivalent von Mises shear strain to investigate the plastic mechanisms in pearlite under cyclic deformation. Fig. 7 shows the atomic strain distribution in both ferrite and cementite phases at different cycle numbers with applied strains of $6.0 \%$ and $9.0 \%$. In Fig. 7a no slip transfers across the ferrite-cementite interface at an applied strain of $6.0 \%$. Almost all the highly strained atoms are in the ferrite phase, which indicates that the main activated slip systems occur in the ferrite phase, while no slip system forms in the cementite phase. Only few highly strained atoms are present in the cementite phase near the ferrite-cementite interface. At an elevated strain level of $9.0 \%$, the slip system in the $(\overline{101})_{\alpha}$ plane in ferrite and $(\overline{103})_{\theta}$ plane in cementite are activated, as shown in Fig. 7b. A slip transfer mechanism across the interface from (a)

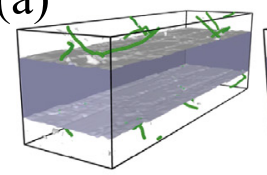

(b)

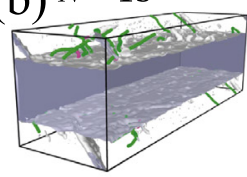

(c) $N=15$

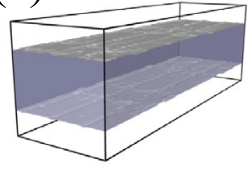

$N=0$

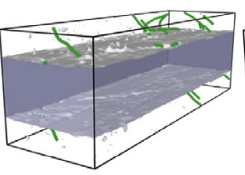

$N=30$

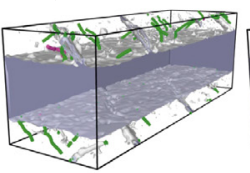

$N=30$

Color Dislocation type

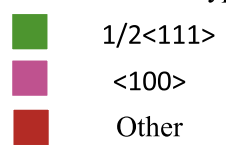

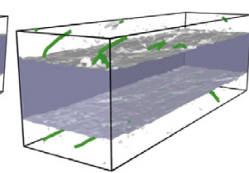

$N=60$

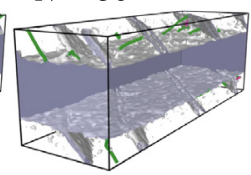

$N=60$

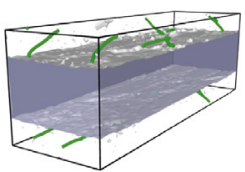

$N=80$

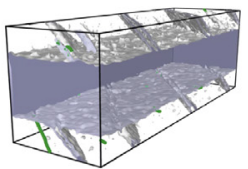

$N=80$

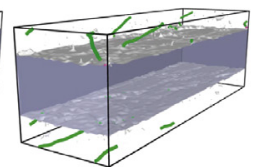

$N=100$

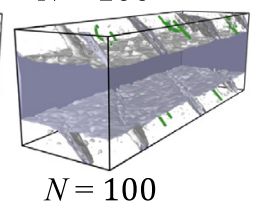

$\underbrace{\mathrm{x}_{\text {Cyclic loading direction }}^{(001)_{\theta} \|(11 \overline{2})_{\alpha}}}_{\mathrm{X}}$

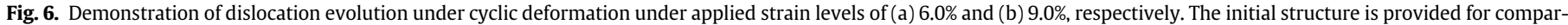
ison in (c). Gray planes in ferrite are the defect meshes. $N$ represents the number of cycles. Dislocation coloring and visualization scheme are the same as in Fig. 5. 

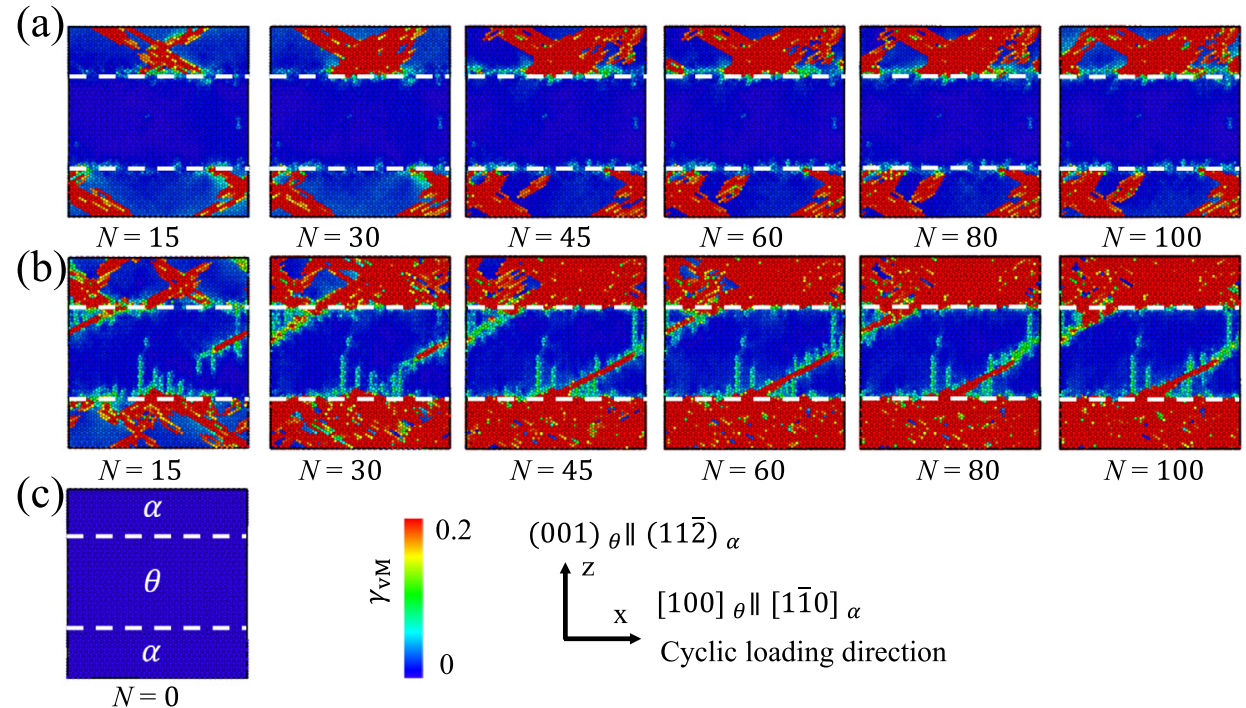

$N=30$
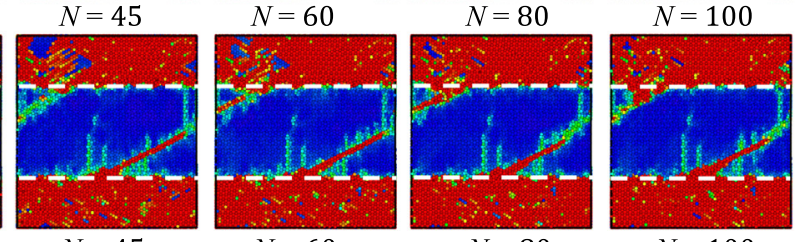

$N=45$

$N=60$

$N=80$

$N=100$

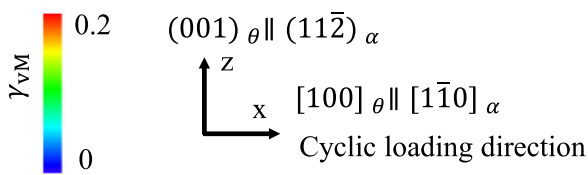

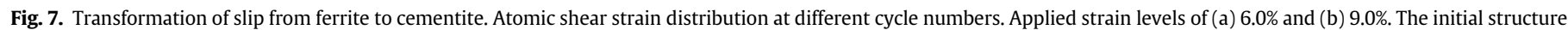
is provided for comparison in (c). White horizontal dashed lines indicate the initial interface between ferrite and cementite in pearlite.

the ferrite to cementite phases is observed, as shown in Supplementary Fig. S2. The result is in agreement with the findings in Ref. [24]. The slip transfer from the ferrite to cementite is a potential fatigue and failure mechanism in cold-drawn pearlitic steels. The slip transfer across the interface from ferrite to cementite induces shear deformation in cementite. As cementite is a brittle phase, this can cause catastrophic failure in the whole sample. The prediction is in good agreement with crystallographic analyses of slip transfer across the ferrite-cementite interface by using the LRB criteria [47-49], as reported by Karkina et al. [11,12]. The onset of slip transfer depends on the magnitude of the plastic strain in pearlite during the cyclic loading. When the plastic strain is higher than critical values, $3.6 \%$ in tension and $2.9 \%$ in compression, slip transfers from the ferrite phase to the cementite phase. The quantitative critical conditions for the slip transfer are shown in Supplementary Table S1 in the Supplementary Material. Under monotonic uniaxial deformation, the sequence of mechanical responses in pearlite is first elastic deformation in both phases, then plastic deformation in the ferrite phase, and eventually slip in the cementite phase [23]. The three loading conditions used in this work therefore mimic the stages of the mechanical response under uniaxial deformation in pearlite.

We quantitatively analyzed the changes in the highly strained atoms in both ferrite and cementite phases to further explore the plastic behavior under cyclic loading. Fig. 8 shows the fraction of atoms with high shear strain, $\gamma_{\mathrm{vM}}>0.3$ [26], as a function of cycle number under applied strains of $6.0 \%$ and $9.0 \%$. The percentage of highly strained atoms in ferrite is much greater than that in cementite. This indicates that most of the slip happens only in the ferrite phase and that the plastic deformation of the pearlite is mainly due to the ferrite phase. At an applied strain of $6.0 \%$, the number of highly strained atoms in pearlite increases in the first 30 cycles and then remains nearly unchanged with increasing cycle number. The number of highly strained atoms in cementite is close to zero during cyclic deformation. The slight difference in the percentage of highly strained atoms between ferrite and pearlite is attributed to atoms in cementite near the ferrite-cementite interface undergoing high shear deformation. At an applied strain of $9.0 \%$, the percentage of highly strained atoms in pearlite increases rapidly in the first 30 cycles and then continues to increase slowly. An obvious increase in the number of highly strained atoms in cementite is caused by the activated slip plane $(\overline{1} 03)_{\theta}$. With increasing cycle numbers, a continuous increase in the percentage of highly strained atoms in cementite occurs, which may be ascribed to the fact the slip planes do not completely fill the entire cementite phase. In other words, the number of highly strained atoms in cementite is still less than the saturation value.

\subsection{Mechanisms of dislocation nucleation and annihilation}

In plastic deformation of metals, interface can act as: (i) sources of plasticity carriers; (ii) sinks for mobile plasticity via absorption and annihilation; (iii) barriers to glissile defect motion; and (iv) storage sites for defects [46]. Pearlite is unique in that the interaction between interfaces and dislocations plays an important role in its cyclic deformation.

Since the mechanisms of dislocation annihilation are different under $6.0 \%$ and $9.0 \%$ straining, we analyze the mechanisms of different strain levels separately. First of all, we chose to conduct an indepth discussion analysis under 6.0\% straining. In Figs. 9 and 10 we show the details of processes of a prototypical dislocation nucleation and annihilation in the first two cycles during cyclic deformation. Only one dislocation is shown in the figures for clarity. Dislocations were analyzed and visualized by CNA as implemented in the OVITO software. The defect atoms in the dislocation core are colored according to their distance from the ferrite-cementite interface. The ferritecementite interface plays an important role in dislocation nucleation and annihilation during cyclic deformation [50,51]. Dislocations nucleate from the ferrite-cementite interface at the yielding point, and then expand towards the opposite interface, as shown in Fig. 9.

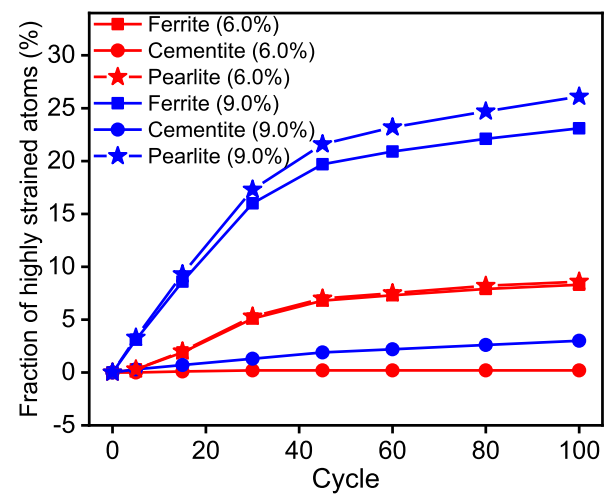

Fig. 8. Fraction of highly strained atoms with atomic shear strain larger than 0.3 as a function of the cycle number in ferrite, cementite, and pearlite under applied strains of $6.0 \%$ and $9.0 \%$. 


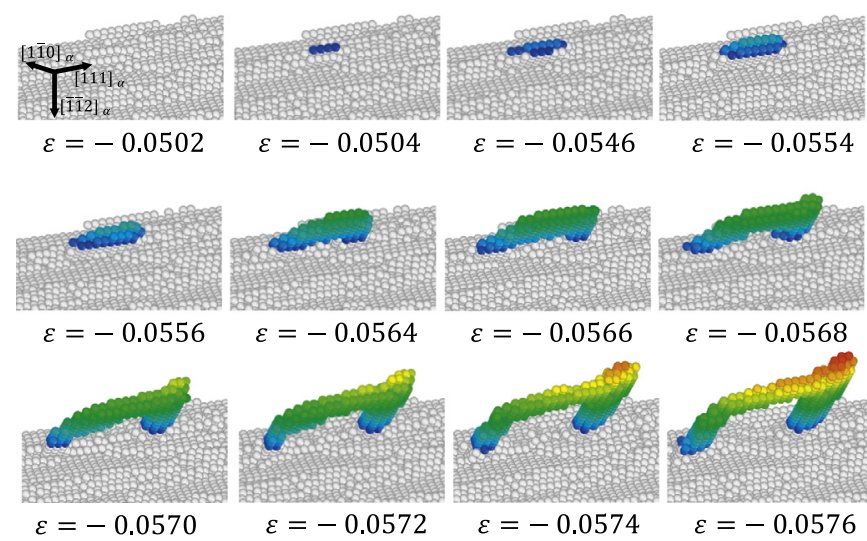

Fig. 9. The nucleation process of a $1 / 2<111>$ dislocation at the ferrite-cementite interface in the first cycle with an applied strain of $6.0 \%$. gray atoms represent cementite. Atoms in color denote defect atoms in the ferrite phase. Dislocations are recognized by CNA and all perfect BCC atoms have been removed for clarity.

Once a dislocation reaches the opposite interface, it becomes a straight screw dislocation. Similar results have been observed in the ferrite-cementite system under tensile deformation [23,24]. When the strain is reversed during cyclic deformation, the nucleated dislocation annihilates at the ferrite-cementite interface and the interface structures recovers, as shown in Fig. 10. Fig. 11a shows that the nucleated dislocation can annihilate completely at interface in the first two cycle with applied strain of $6.0 \%$. In Fig. 11b it shows that some dislocations, which do not connect the two interfaces, are blocked by the residual strains in the ferrite phase after 12 cycles. The residual strains accumulated in the ferrite phase are denoted by the defect meshes as shown in Fig. 11, which are introduced by the cyclic deformations. At the applied strain of $6.0 \%$, therefore, the prevalent mechanism is that dislocations nucleate and then annihilate at the same interface in the first 12 cycles. After 12 cycles, residual strain cloud in the ferrite phase results in residual stress, which impedes the complete annihilation of dislocations at interface.

In the case of the applied strain of $9.0 \%$, Fig. 11c shows different dislocation behaviors. There are two types of dislocations that are being blocked by the residual strain patterns accumulated in the ferrite phase in the first two cycles. One type of the dislocations connects the two interfaces, but the other type of dislocations does not connect the two interfaces. As a result, dislocations cannot annihilate

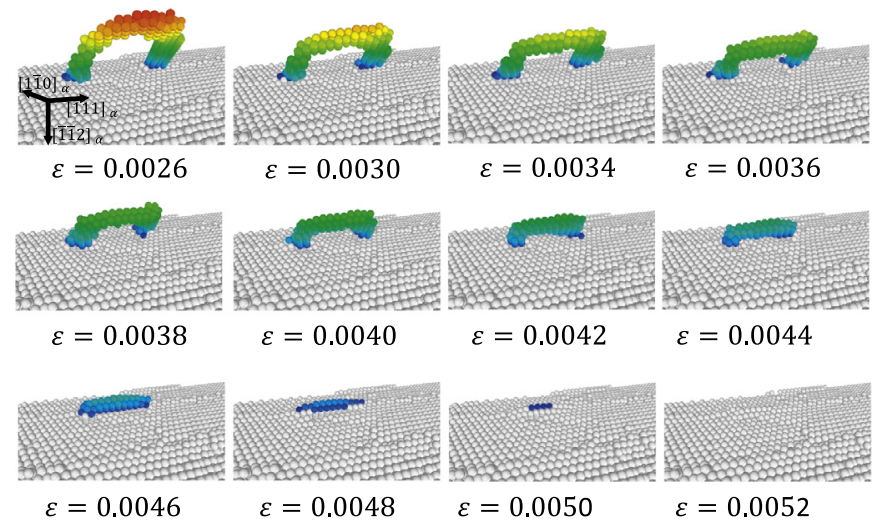

Fig. 10. The annihilation process of a $1 / 2<111>$ dislocation at the ferrite-cementite interface in the second cycle with an applied strain of $6.0 \%$. Atom coloring and dislocation visualization scheme are the same as in Fig. 9. completely during the subsequent cyclic loadings. Different from the applied strain of $6.0 \%$, the main mechanism under higher strain level (i.e., 9.0\%) is a new scenario. The residual strain cloud already appears in ferrite even in the first two cycles. The resulted residual stress in ferrite thus may hinder the complete annihilation of dislocations at interface. This mechanism is similar to that happens under the applied strain of $6.0 \%$ after 12 cycles.

As Fig. 9 shows, it takes about 6.2 ps for a dislocation embryo to form a nucleated dislocation, which is corresponding to the strain magnitude from $\varepsilon=-5.02 \%$ to $\varepsilon=-5.64 \%$. In contrast, Fig. 10 shows that the dislocation annihilation process experiences shorter strain interval, i.e., from the strain magnitude $0.46 \%$ to $0.52 \%$, which means $0.6 \mathrm{ps}$ in the present simulation scheme. Therefore, the dislocation annihilation is also a thermally activated process but with smaller activation barrier than that of a dislocation nucleation process [52]. Note that the low temperature and high strain rate (high stress) conditions applied in the present simulations of cyclic deformation will boost the displacive mechanisms, such as dislocation nucleation and annihilation processes at the interface. However, this option will definitely suppress the diffusive mechanisms, e.g. the dissociation and migration of carbon atoms from cementite to ferrite, which may miss the subsequent mechanism of carbon-dislocation interaction [7]. If the temperature is higher, like the condition at room temperature, the thermally-activated dislocation nucleation process will be encouraged, while the dislocation annihilation process which is of smaller activation barrier is quicker, and it follows the pace of dislocation nucleation. Therefore, it is anticipated that the steady-state cyclic deformation with constant dislocation density become earlier with increasing temperature.

\subsection{Rationalization of the fatigue mechanism of pearlite}

The deformation mechanism of pearlite under cyclic deformation is ascribed to the synergistic effects between the ferrite and the cementite phases. The plasticity of pearlite is largely governed by the mechanisms operating in the ferrite phase during cyclic deformation (shown in Fig. 8). At a low strain level (4.0\%), elastic deformation occurs in pearlite and no dislocation generates at the interface within the number of deformation cycles studied. At an intermediate strain level (6.0\%), however, plastic deformation of pearlite begins to thanks to the dislocation behaviors operating only in the ferrite phase. At a higher strain level (9.0\%), high stress drives plastic and inelastic mechanisms in ferrite and cementite, respectively, and eventually dislocation slip transfers across the interface from the ferrite to cementite phases.

The underlying mechanisms of the nanoscale ferrite-cementite lamellae under cyclic deformation are related to the interplay between the ferrite-cementite interface and the interfacial dislocation. The interface plays multiple roles, serving as a source for nucleation, absorption, and annihilation of dislocations. The plastic mechanisms of dislocation nucleation and annihilation are both observed in our simulations of the cyclic plastic deformation of pearlite (shown in Figs. 9-11). After onset of plasticity, dislocations nucleate at the ferrite-cementite interface and spread through the whole ferrite phase (shown in Fig. 9). The nucleated dislocations expand until they reach the opposite side of the interface. The lattice structures are different on the two sides of the ferrite-cementite interface, i.e., ferrite is a BCC crystal while cementite is an orthorhombic crystal. Compared with the dislocations existing in ferrite, the dislocation density in cementite is lower because it is difficult to deform plastically due to the nature of covalent bonding $[44,53]$. The unique interface structure prevents dislocations in ferrite from passing across the interface, and some of them are absorbed at the ferrite-cementite interface. Nucleated dislocations become straight screw-type dislocations in ferrite once they reach the interface again. At applied strain of $6.0 \%$, when the sign of the applied strain is reversed due to cyclic 
(a)

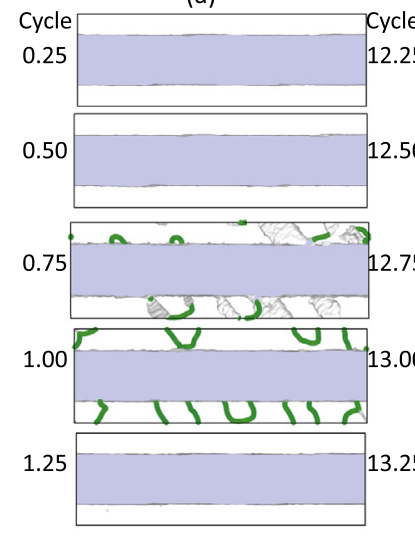

(b)
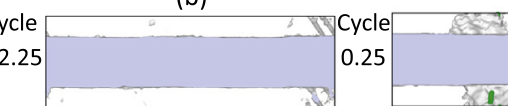

(c)
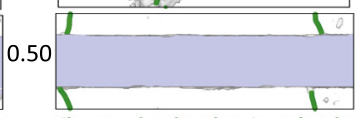

0.75

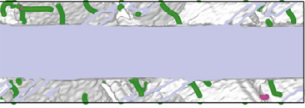

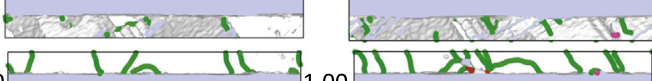
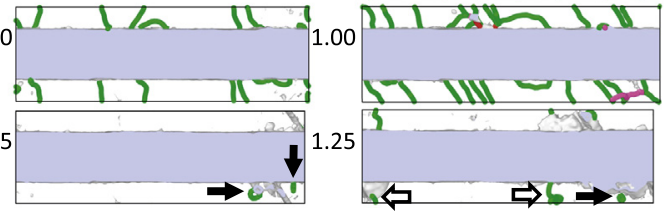

Color Dislocation type
$1 / 2<111>$
$<100>$
Other

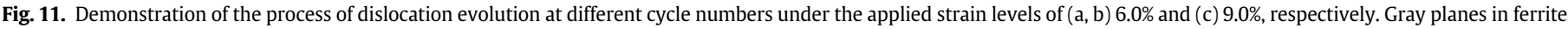

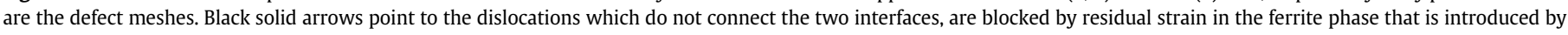

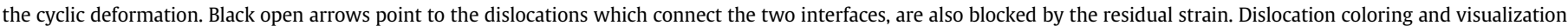
scheme are the same as in Fig. 5.

loading, the dislocations annihilate completely at the ferrite-cementite interface in the first 12 cycles (shown in Figs. 10 and 11a). With an increase in cycle number, residual stress in ferrite blocks dislocations and impede their complete annihilation at interface. As applied strain increases (9.0\%), dislocations do not annihilate fully from the beginning to the steady state during cyclic loading.

There is a competitive relationship between dislocation nucleation and annihilation during cyclic deformation. This explains the appearance of steady-state flow after several tens of cycles. At high strain levels (6.0\% and 9.0\%), the dislocation density in ferrite increases rapidly in the first few cycles (shown in Fig. 4b). This indicates a rapid accumulation of dislocations in ferrite after cyclic plastic deformation because the dislocation nucleation rate is greater than the rate of dislocation annihilation. More dislocation nucleation is activated from the interface because of the high stress level in the first few cycles, as shown in Fig. 3. With the number of cycles increases, the peak stress decreases and therefore dislocation nucleation rate decreases. However, the rate of dislocation annihilation is a quicker process with activation barrier smaller than that of dislocation nucleation. The rate of dislocation annihilation increases with increasing cycle numbers, since there will more nucleated dislocations pile up at the opposite interface as sources of dislocation annihilation. Therefore, it leads to a decrease in dislocation density in the ferrite phase. When the rate of dislocation nucleation is close to that of dislocation annihilation, a steady state forms with constant dislocation density and peak stress magnitudes, as shown in Figs. 4b and 3, respectively. In addition, a straightforward quantitative examination of the rates of dislocation nucleation and annihilation during cyclic loading, respectively, deserves furthermore study in the future work.

The three most prominent slip planes in the cementite phase are (001), (010), and (100) at room temperature [45,54,55]. However, the geometry of the interface makes ductility impossible because the three possible slip directions [001], [010], and [100] are mutually perpendicular [44]. Covalent bonding in cementite makes it brittle and hard at room temperature [43]. After the onset of plasticity, nucleated dislocations at the interface slip until they reach the opposite side of the interface, where they are absorbed into the cementite phase. Once the magnitude of plastic strain in pearlite is higher than critical values, $3.6 \%$ in tension and $2.9 \%$ in compression, slip will transfer from ferrite to cementite to accommodate strains accumulated in the cyclic deformation. The dislocations absorbed on the interface may then induce a local shear deformation in the cementite phase [26]. This was also seen in our simulations, as shown in Fig. 7. Since the cementite is brittle, the cumulative shear deformation in cementite (shown in Fig. 8) perhaps lead to the nucleus of short cracks at the interface with increasing cyclic deformation, causing a fatigue failure.
A crack will propagate suddenly upon reaching a critical size, and the structure will fracture catastrophically, resulting in material failure $[2,42,56]$. In actual application, the number of dislocation has been found to initially increase and then saturate during fatigue in pearlitic steels [7], polycrystalline carbon steels [5], and stainless steels [57-59]. Our simulations give an atomic-scale view of dislocation nucleation and annihilation during cyclic deformation.

\section{Conclusion}

The atomistic simulations in this work characterize the mechanical response and the corresponding dislocation mechanism of the ferrite-cementite interface with Bagaryatskii OR in pearlite under cyclic deformation. Three different strain levels (4.0\%, 6.0\%, and 9.0\%) were chosen to mimic the mechanical response of pearlite under fatigue deformation. At the lowest strain level (4.0\%), the ferrite-cementite interface deforms elastically with constant peak stress amplitude during cyclic deformation. As the strain level increases (6.0\%), the maximum stress remains unchanged because of elastic tension, while the minimum stress drops because of strain softening. At a still higher strain level (9.0\%), the maximum and minimum stresses drop because dislocations nucleate at the interface during both compressive and tensile stages. When the applied strain level is $6.0 \%$, the plasticity in pearlite occurs almost completely in ferrite. At a high strain level of $9.0 \%$, most of the highly strained atoms in pearlite are in the ferrite phase, but a few atoms in cementite have high shear strains (atomic strains greater than 0.3), and slip transfer occurs across the interface from the ferrite phase to the cementite phase once the magnitude of plastic strain in pearlite is higher than critical values.

The micro-mechanism underlying cyclic plastic deformation (at strain levels of $6.0 \%$ and $9.0 \%$ ) in pearlite is governed by the interplay between the interface and the dislocations. Interfaces play multiple roles in deformation, providing sites for the nucleation, absorption, and annihilation of dislocations. A competitive relationship between dislocation nucleation and dislocation annihilation exists during cyclic plastic deformation. Dislocation density in ferrite increases rapidly in the first 30 cycles, which indicates rapid dislocation accumulation. As the number of cycles increases, dislocation density in ferrite decreases because the rate of dislocation annihilation increases, while the rate of dislocation nucleation decreases. When the dislocation nucleation rate is equal to that of dislocation annihilation, the dislocation density in ferrite remains stable and steady-state flow occurs. However, an important implication of our simulations is that plasticity transfer from ferrite to cementite can occur when dislocations cross the interface. This is dangerous for pearlite because the plasticity accumulation in brittle cementite phase will result in 
catastrophic material failure under cyclic deformation. Our simulations thus reveal a possible failure mechanism of pearlite steel under real fatigue deformation.

\section{Declaration of Competing Interest}

The authors declare that they have no known competing financial interests or personal relationships that could have appeared to influence the work reported in this paper.

\section{Acknowledgments}

This work was financially supported by the National Key Research and Development Program of China (No. 2017 YFB0702003 and No. 2017YFB0701502), the NSFC (No. 11790292, No. 11572324, and No. 11672299), the Strategic Priority Research Program (No. XDB22040302 and No. XDB22040303), the Key Research Program of Frontier Sciences (No. QYZDJSSW-JSC011), and the Youth Innovation Promotion Association (No. 2017025) of the Chinese Academy of Sciences. All the calculations were performed on the "Era" petascale supercomputer of the Computer Network Information Center of CAS.

\section{Supplementary materials}

Supplementary material associated with this article can be found in the online version at doi:10.1016/j.actamat.2019.12.052.

\section{References}

[1] C. Borchers, R. Kirchheim, Cold-drawn pearlitic steel wires, Prog. Mater. Sci. 82 (2016) 405-444.

[2] W.D. Callister, D.G. Rethwisch, Materials Science and Engineering: An Introduction, John Wiley \& Sons, New York, 2007.

[3] Q. Pan, H. Zhou, Q. Lu, H. Gao, L. Lu, History-independent cyclic response of nanotwinned metals, Nature 551 (7679) (2017) 214.

[4] C. Gaudin, X. Feaugas, Cyclic creep process in AISI 316L stainless steel in terms of dislocation patterns and internal stresses, Acta Mater. 52 (10) (2004) 3097-3110.

[5] G. Kang, Y. Dong, Y. Liu, H. Wang, X. Cheng, Uniaxial ratchetting of 20 carbon steel: macroscopic and microscopic experimental observations, Mater. Sci. Eng. A 528 (16) (2011) 5610-5620.

[6] G. Kang, Y. Dong, H. Wang, Y. Liu, X. Cheng, Dislocation evolution in 316L stainless steel subjected to uniaxial ratchetting deformation, Mater. Sci. Eng. A 527 (21) (2010) 5952-5961.

[7] L. Liang, L. Xiang, Y. Wang, Y. Chen, H. Wang, L. Dai, Ratchetting in cold-drawn pearlitic steel wires, Metall. Mater. Trans. A 50 (10) (2019) 4561-4568.

[8] T. Furuhara, T. Maki, Grain boundary engineering for superplasticity in steels, J. Mater. Sci. 40 (4) (2005) 919-926.

[9] Y.J. Zhang, G. Miyamoto, K. Shinbo, T. Furuhara, Quantitative measurements of phase equilibria at migrating $\alpha / \gamma$ interface and dispersion of VC interphase precipitates: evaluation of driving force for interphase precipitation, Acta Mater. 128 (2017) 166-175.

[10] H. Dong, H. Chen, W. Wang, Y. Zhang, G. Miyamoto, T. Furuhara, et al., Analysis of the interaction between moving $\alpha / \gamma$ interfaces and interphase precipitated carbides during cyclic phase transformations in a Nb-containing Fe-C-Mn alloy, Acta Mater. 158 (2018) 167-179.

[11] L.E. Kar'kina, I.G. Kabanova, I.N. Kar'kin, Strain transfer across the ferrite/cementite interface in carbon steels with coarse lamellar pearlite, Phys. Met. Metall. 119 (11) (2018) 1114-1119.

[12] L. Karkina, I. Karkin, I. Kabanova, A. Kuznetsov, Crystallographic analysis of slip transfer mechanisms across the ferrite/cementite interface in carbon steels with fine lamellar structure, J. Appl. Crystallogr. 48 (1) (2015) 97-106.

[13] S. Tagashira, K. Sakai, T. Furuhara, T. Maki, Deformation microstructure and tensile strength of cold rolled pearlitic steel sheets, ISIJ Int. 40 (11) (2000) 11491156.

[14] M.A. Phillips, B.M. Clemens, W.D. Nix, A model for dislocation behavior during deformation of Al/Al3Sc (fcc/L12) metallic multilayers, Acta Mater. 51 (11) (2003) 3157-3170.

[15] G. Langford, Deformation of pearlite, Metall. Trans. A 8 (6) (1977) 861-875.

[16] J.D. Embury, R.M. Fisher, The structure and properties of drawn pearlite, Acta Metall. 14 (2) (1966) 147-159.

[17] M. Guziewski, S.P. Coleman, C.R. Weinberger, Interface energetics and structure of the pearlitic microstructure in steels: an atomistic and continuum investigation, Acta Mater. 155 (2018) 1-11.

[18] Y.T. Zhou, S.J. Zheng, Y.X. Jiang, T.Z. Zhao, Y.J. Wang, X.L. Ma, Atomic structure of the $\mathrm{Fe} / \mathrm{Fe} 3 \mathrm{C}$ interface with the Isaichev orientation in pearlite, Philos. Mag. 97 (2017) 1-12.
[19] M. Guziewski, S.P. Coleman, C.R. Weinberger, Atomistic investigation into the atomic structure and energetics of the ferrite-cementite interface: the Bagaryatskii orientation, Acta Mater. 119 (2016) 184-192.

[20] Y. Bagaryatskii, G. Nosova, T. Tagunova, O. kristallicheskoi, Strukture i prirode omegafazy v splavakh titana s khromom, Dokl. Akad. Nauk SSSR 105 (6) (1955) 1225.

[21] J. Kim, K. Kang, S. Ryu, Characterization of the misfit dislocations at the ferrite cementite interface in pearlitic steel: an atomistic simulation study, Int. J. Plast. 83 (2016) 302-312

[22] M. Guziewski, S.P. Coleman, C.R. Weinberger, Atomistic investigation into interfacial effects on the plastic response and deformation mechanisms of the pearlitic microstructure, Acta Mater. 180 (2019) 287-300.

[23] M. Guziewski, S.P. Coleman, C.R. Weinberger, Atomistic investigation into the mechanical properties of the ferrite-cementite interface: the Bagaryatskii orientation, Acta Mater. 144 (2018) 656-665.

[24] T. Shimokawa, T. Niiyama, M. Okabe, J. Sawakoshi, Interfacial-dislocation-controlled deformation and fracture in nanolayered composites: toward higher ductility of drawn pearlite, Acta Mater. 164 (2019) 602-617.

[25] L. Xiang, L.W. Liang, Y.J. Wang, Y. Chen, H.Y. Wang, L.H. Dai, One-step annealing optimizes strength-ductility tradeoff in pearlitic steel wires, Mater. Sci. Eng. A 757 (2019) 1-13.

[26] H. Ghaffarian, A.K. Taheri, K. Kang, S. Ryu, Molecular dynamics simulation study on the effect of the loading direction on the deformation mechanism of pearlite, Multiscale Sci. Eng. 1 (1) (2019) 47-55.

[27] S. Plimpton, Fast parallel algorithms for short-range molecular dynamics, J. Comput. Phys. 117 (1) (1995) 1-19.

[28] K. Henriksson, C. Björkas, K. Nordlund, Atomistic simulations of stainless steels: a many-body potential for the $\mathrm{Fe}-\mathrm{Cr}-\mathrm{C}$ system, J. Phys. Condens. Matter 25 (44) (2013) 445401

[29] Y. Zhang, J. Budnick, F. Sanchez, W. Hines, D. Yang, J. Livingston, NMR studies in orthorhombic Fe3B1 - x C x (0.1 x x 0.4), J. Appl. Phys. 61 (8) (1987) 4358-4360

[30] J.P. Hirth, J. Lothe, T. Mura, Theory of dislocations, J. Appl. Mech. 50 (1983) 476.

[31] S. Nosé, A molecular dynamics method for simulations in the canonical ensemble, Mol. Phys. 52 (2) (1984) 255-268.

[32] W.G. Hoover, Canonical dynamics: equilibrium phase-space distributions, Phys Rev. A 31 (3) (1985) 1695.

[33] M. Parrinello, A. Rahman, Polymorphic transitions in single crystals: a new molecular dynamics method, J. Appl. Phys. 52 (12) (1981) 7182-7190.

[34] J. Languillaume, G. Kapelski, B. Baudelet, Cementite dissolution in heavily cold drawn pearlitic steel wires, Acta Mater. 45 (3) (1997) 1201-1212.

[35] A. Stukowski, Visualization and analysis of atomistic simulation data with OVITO-the open visualization tool, Model. Simul. Mater. Sci. Eng. 18 (1) (2009) 015012.

[36] A. Stukowski, V.V. Bulatov, A. Arsenlis, Automated identification and indexing of dislocations in crystal interfaces, Model. Simul. Mater. Sci. Eng. 20 (8) (2012) 085007

[37] J.D. Honeycutt, H.C. Andersen, Molecular dynamics study of melting and freezing of small Lennard-Jones clusters, J. Phys. Chem. 91 (19) (1987) 4950-4963.

[38] M.L. Falk, J.S. Langer, Dynamics of viscoplastic deformation in amorphous solids, Phys. Rev. E 57 (6) (1998) 7192-7205.

[39] F. Shimizu, S. Ogata, J. Li, Theory of shear banding in metallic glasses and molecular dynamics calculations, Mater. trans. (2007) 0710160231.

[40] J. Schijve, Fatigue of Structures and Materials, Springer Science \& Business Media, 2001.

[41] X. Yuan, W. Yu, S. Fu, D. Yu, X. Chen, Effect of mean stress and ratcheting strain on the low cycle fatigue behavior of a wrought 316LN stainless steel, Mater. Sci. Eng. A 677 (2016) 193-202.

[42] M.A. Meyers, A. Mishra, D.J. Benson, Mechanical properties of nanocrystalline materials, Prog. Mater. Sci. 51 (4) (2006) 427-556.

[43] T. Terashima, Y. Tomota, M. Isaka, T. Suzuki, M. Umemoto, Y. Todaka, Strength and deformation behavior of bulky cementite synthesized by mechanical milling and plasma-sintering, Scr. Mater. 54 (11) (2006) 1925-1929.

[44] J.G. Sevillano, Room temperature plastic deformation of pearlitic cementite, Mater. Sci. Eng. 21 (1975) 221-225.

[45] A. Keh, Imperfections and plastic deformation of cementite in steel, Acta Metall. 11 (9) (1963) 1101-1103.

[46] J. Wang, A. Misra, An overview of interface-dominated deformation mechanisms in metallic multilayers, Curr. Opin. Solid State Mater. Sci. 15 (1) (2011) 20-28.

[47] T.C. Lee, I.M. Robertson, H.K. Birnbaum, Prediction of slip transfer mechanisms across grain boundaries, Scr. Metall. 23 (5) (1989) 799-803.

[48] T. Lee, I. Robertson, H. Birnbaum, TEM in situ deformation study of the interaction of lattice dislocations with grain boundaries in metals, Philos. Mag. A 62 (1) (1990) 131-153.

[49] T.C. Lee, I.M. Robertson, H.K. Birnbaum, An in situ transmission electron microscope deformation study of the slip transfer mechanisms in metals, Metall. Trans. A 21 (9) (1990) 2437-2447.

[50] J. Embury, J. Hirth, On dislocation storage and the mechanical response of fine scale microstructures, Acta Metall. Mater. 42 (6) (1994) 2051-2056.

[51] V. Gavriljuk, Comment on "Effect of interlamellar spacing on cementite dissolution during wire drawing of pearlitic steel wires", Scr. Mater. 45 (12) (2001) 1469-1472.

[52] A. Evans, R. Rawlings, The thermally activated deformation of crystalline materials, Phys. Stat. Sol. 34 (1) (1969) 9-31.

[53] A. Inoue, T. Ogura, T. Masumoto, Burgers vectors of dislocations in cementite crystal, Scr. Metall. 11 (1) (1977) 1-5.

[54] A. Inoue, T. Ogura, T. Masumoto, Microstructures of deformation and fracture of cementite in pearlitic carbon steels strained at various temperatures, Metall. Trans. A 8 (11) (1977) 1689-1695. 
[55] D. Porter, K. Easterling, G. Smith, Dynamic studies of the tensile deformation and fracture of pearlite, Acta Metall. 26 (9) (1978) 1405-1422.

[56] M.D. Sangid, The physics of fatigue crack initiation, Int. J. Fatigue 57 (2013) 58-72.

[57] K. Dutta, R. Kishor, L. Sahu, A. Mondal, On the role of dislocation characters influencing ratcheting deformation of austenitic stainless steel, Mater. Sci. Eng. A 660 (2016) 47-51.
[58] R. Kishor, L. Sahu, K. Dutta, A. Mondal, Assessment of dislocation density in asymmetrically cyclic loaded non-conventional stainless steel using X-ray diffraction profile analysis, Mater. Sci. Eng. A 598 (2014) 299-303.

[59] G. Kang, Y. Dong, H. Wang, Y. Liu, X. Cheng, Dislocation evolution in 316L stainless steel subjected to uniaxial ratchetting deformation, Mater. Sci. Eng. A 527 (21-22) (2010) 5952-5961. 